Communications in Physics, Vol.21, No. 2 (2011), pp. 97-104

\title{
STUDY OF PHYSICAL PROPERTIES OF COMPLEX SCALAR FIELD AT FINITE DENSITY
}

\author{
TRAN HUU PHAT \\ Vietnam Atomic Energy Commission \\ BUI THI PHUONG THUY \\ Faculty of Physics, Hanoi University of Education
}

\begin{abstract}
The physical properties of a relativistic model for complex scalar field at finite density are studied. It is shown that the boson is condensed when the critical chemical potential is equal to the boson mass in vacuum and the system undergoes the phase transition from normal to superconducting states. The latter state is signaled by the manifestations of the Meissner effect and the Abrikosov vortices in the presence of an electromagnetic field. Moreover, the boson condensate is separated by a topologically stable domain wall.
\end{abstract}

\section{INTRODUCTION}

It is known that the evolution of modern physics always links closely with scalar fields. They appear in a lot of physical theories. Scalar fields play the role of order parameters in the Landau theory of phase transition [1], in the Ginzburg-Landau theory of superconductivity [2]. Fundamental scalar fields are necessarily present in all unification theories: from the Standard Model of particles to the Grand Unification Model [3,4], here scalar fields describe the Higgs bosons. In superstring theory scalar field emerges in the form of dilaton and in Cosmology scalar fields are needed in the inflation theory $[5,6]$. However, all the above mentioned theories of scalar fields have been considered in vacuum only. It is expected that in medium these fields manifest many other interesting features which are absent in vacuum. In effect, it is well known that the condensation of kaons and charged pions in dense matter and compact stars has been the interesting subject since long ago [7-10]. Bose-condensed states are expected to be found in the interior of compact stars with density around $3 \rho_{0}$, where $\rho_{0}$ is normal nuclear density. Until now we are aware of only few points in the $T-\rho$ plane, namely, the vacuum $(T=0, \rho=0)$ and nuclear matter $\left(T=0, \rho=\rho_{0}=0.17 \mathrm{fm}^{-3}\right)$. Therefore, there are much experimental and theoretical efforts to get information about other regions. In recent years experimental studies are carried out through observing the matter produced in heavy-ion collisions at intermediate energies, in particular, the nuclear reactions induced by radioactive beams which offer a new opportunity to consider the isospin degree of freedom of asymmetric nuclear matter. As a consequence, the theoretical researches of Quantum Chromodynamics (QCD) at finite baryon density and isospin chemical potential are intensively implemented. It was shown that in the color-flavor-locked phase kaon condensation occurs at high baryon density and low temperature [11] and the lattice simulation of QCD at finite isospin chemical potential 
[12-14] proved that there manifests the Bose-Einstein condensation of charged pions at high isospin density and low temperature. In this connection, investigating simplified models of strongly interacting matter, such as the Nambu-Jona-Lasinio model [15-18], the linear sigma model [19-21], the chiral perturbation theory [22-25] etc., is very important for the understanding of matter states under different conditions. In this respect, in this paper we focus on studying a simple relativistic model of complex scalar fields at finite density and zero temperature, whose Lagrangian reads:

$$
\begin{aligned}
L & =\left(\partial_{\alpha}+i \mu \delta_{0 \alpha}\right) \phi^{*}\left(\partial^{\alpha}-i \mu \delta_{0 \alpha}\right) \phi-V_{0} \\
V_{0} & =m^{2} \phi^{*} \phi+\frac{\lambda}{2}\left(\phi^{*} \phi\right)^{2} .
\end{aligned}
$$

in which $\mu$ is the chemical potential.

This paper is organized as follows. In Section II the physical properties of the system are studied in detail.The conclusion and outlook are given in Section III.

\section{PHYSICAL PROPERTIES}

\section{Spontaneous symmetry breaking and Goldstone theorem}

Equation (1) can be rewritten more explicitly,

$$
\begin{aligned}
& L=\partial_{\alpha} \phi^{*} \partial^{\alpha} \phi+i \mu\left(\phi^{*} \partial_{0} \phi-\phi \partial_{0} \phi^{*}\right)-V \\
& V=\left(m^{2}-\mu^{2}\right) \phi^{*} \phi+\frac{\lambda}{2}\left(\phi^{*} \phi\right)^{2}
\end{aligned}
$$

Suppose that $\phi$ develops the expectation value $u$ in the ground state and in the tree approximation it is determined by the minimum of $V$

$$
\left(m^{2}-\mu^{2}\right) u+\lambda u^{3}=0
$$

which produces a vanishing solution $\mathrm{u}=0$ and two non-trivial solutions

$$
u_{ \pm}= \pm \sqrt{\frac{\mu^{2}-m^{2}}{\lambda}}
$$

Eq. (4) tells that $\mathrm{u}$ is real only if $\mu^{2}>m^{2}$.

Hence, the ground state $M$ is degenerate, consisting of two disconnected points $u_{+}$, and, $u_{-}$. It is conventional to choose the vacuum state corresponding to $u_{+}$, then the symmetry group $U(1)$ is spontaneously broken. Physically, this means that the boson is condensed in a sufficiently dense matter and $u^{2}$ is exactly the density of the boson condensate. It is worth to note that Eq. (3) can be understood as the minimum condition for $\phi=u e^{i \alpha}$ with $u=u_{+}$. So, in reality the ground state $\mathrm{M}$ is a circle $S^{1}$. Next let us check whether the Goldstone theorem is preserved or not. For this goal, let us expand

$$
\begin{aligned}
\phi & =u+\phi_{1}+i \phi_{2}, \\
\phi^{*} & =u+\phi_{1}-i \phi_{2} .
\end{aligned}
$$

Here for simplicity the symbol $\mathrm{u}$ is used to denote $u_{+}$from now on. 
Inserting (5) into Eqs. (2) it is obtained the new expression for the Lagrangian:

$$
\begin{aligned}
L= & \sum_{i=1,2}\left(\partial_{\alpha} \phi_{i}\right)^{2}-2 \mu\left(\phi_{1} \partial_{0} \phi_{2}-\phi_{2} \partial_{0} \phi_{1}\right)+\left(\mu^{2}-m^{2}-3 \lambda u^{2}\right) \phi_{1}^{2}+\left(\mu^{2}-m^{2}-\lambda u^{2}\right) \phi_{2}^{2} \\
& -\frac{\lambda}{2}\left(\phi_{1}^{4}+\phi_{2}^{4}+2 \phi_{1}^{2} \phi_{2}^{2}+4 u \phi_{1}^{3}+4 u \phi_{1} \phi_{2}^{2}-u^{4}\right)
\end{aligned}
$$

which gives immediately the inverse propagator at tree level

$$
\begin{array}{r}
i D^{-1}(\omega, \vec{k})=\left(\begin{array}{ll}
A_{11} & A_{12} \\
A_{21} & A_{22}
\end{array}\right), \\
A_{11}=\omega^{2}-\vec{k}^{2}+\mu^{2}-m^{2}-3 \lambda u^{2}, \\
A_{12}=-A_{21}=2 i \mu \omega, \\
A_{22}=\omega^{2}-\vec{k}^{2}+\mu^{2}-m^{2}-\lambda u^{2} .
\end{array}
$$

Taking into account Eq.(2a) $A_{11}$ and $A_{22}$ are simplified to

$$
A_{11}=\omega^{2}-\vec{k}^{2}-2 \lambda u^{2}, A_{22}=\omega^{2}-\vec{k}^{2} .
$$

we deduce

$$
i D^{-1}(\omega, \vec{k})=\left(\begin{array}{ll}
\omega^{2}-\vec{k}^{2}-2 \lambda u^{2} & 2 i \mu \omega \\
-2 i \mu \omega & \omega^{2}-\vec{k}^{2}
\end{array}\right)
$$

Then the spectrum is defined by (4), namely, $\operatorname{det} D^{-1}(k)=0$ yielding a gapless mode

$$
\omega_{2} \approx \sqrt{\frac{\lambda u^{2}}{\lambda u^{2}+2 \mu^{2}}}|\vec{k}| \stackrel{\vec{k} \rightarrow 0}{\longrightarrow} 0 .
$$

Hence the Goldstone theorem is realized in broken phase. Due to the criteria for superfluidity of Landau [1] the condensate becomes superfluid in broken phase and the speed of sound in the condensate is given by

$$
C=\sqrt{\frac{\lambda u^{2}}{\lambda u^{2}+2 \mu^{2}}}
$$

\section{Meissner Effect}

Let us now consider the system in the external electromagnetic field. The Lagrangian (2) is replaced by:

$$
L=\left(\partial_{0}+i \mu+i e A_{0}\right) \phi^{*}\left(\partial_{0}-i \mu-i e A_{0}\right) \phi-(\nabla+i e \vec{A}) \phi^{*}(\nabla-i e \vec{A}) \phi-V-\frac{1}{4} F^{\nu \mu} F_{\mu \nu}
$$

For $\mu>m$ the $\mathrm{U}(1)$ symmetry is spontaneously broken and let $u^{2}$ be the density of the boson condensate. We decompose

$$
\phi=u+\chi, \phi^{*}=u+\chi^{*}
$$


Substituting (8) into (7) and then varying the resulting Lagrangian with respect to the electromagnetic potentials $A_{\mu}$ we arrive at the equations of the electromagnetic field in the condensate medium

$$
\left(-e^{2} u^{2}\right) A_{\mu}=j_{\mu}
$$

in which $j_{\mu}$ is the supercurrent,

$$
j_{\mu}=i e\left(\chi \partial_{\mu} \chi^{*}-\chi^{*} \partial_{\mu} \chi\right)+i e u\left(\partial_{\mu} \chi^{*}-\partial_{\mu} \chi\right)-e^{2} u\left(\chi^{*}+\chi\right) A_{\mu}-e^{2} \chi^{*} \chi A_{\mu}
$$

Eqs.(9) proves that the photon can only propagates inside the condensate to a depth $l \approx \frac{\hbar}{m_{p h} . c}$, here $m_{p h}$ is the mass of photon, $m_{p h}=e u$. This is the Meissner effect which indicates that the boson condensate is a superconductor. It is very interesting to remark that the superconducting state occurs only when $\mu>\mu_{\text {critic }}=m$.

\section{Abrikosov Vortices}

To proceed to the magnetic vortices emerging in the presence of electromagnetic field we look for the radially symmetric solutions with finite energy per unit length in the cylindrical coordinates $(\rho, \theta, z)$. For convenience let us choose the gauge condition $A_{0}=0$ and consider the static case when $\phi$ is time independent, we have then

$$
E / L=\int d x d y\left\{\left[\left(D_{k} \phi\right)^{*}\left(D_{k} \phi\right)+\frac{\lambda}{2}\left(\phi^{*} \phi-u^{2}\right)^{2}\right]+\frac{\vec{B}^{2}}{8 \pi^{2}}\right\}
$$

where $D_{k}=\partial_{k}-i e A_{k}, k=1,2, \vec{B}=\operatorname{curl} \vec{A}$.

In order for (10) to be finite we ask that when $\rho=R \rightarrow \infty$

$$
\begin{aligned}
|\phi| & \rightarrow u, \\
\left|D_{k} \phi\right| & \rightarrow 0 .
\end{aligned}
$$

It is evident that (11) establishes the map from $M_{\infty}\{\rho=R \rightarrow \infty\}$ to the ground state manifold M, that is the map: $S^{1} \rightarrow S^{1}$ that possesses non-trivial group $\pi_{1}\left(S^{1}\right), \pi_{1}\left(S^{1}\right)=Z$. Accordingly, there are an infinite number of vortices, which are called the Abrikosov vortices. Starting from Eqs.(9) we accept the following ansatz

$$
\begin{aligned}
\phi & =u f(\rho) e^{i n \theta(x),} \\
A_{\mu} & =\frac{n}{e} a(\rho) \partial_{\mu} \theta(x) .
\end{aligned}
$$

The boundary conditions for (13) usually read

$$
\begin{gathered}
f(0)=a(0)=0, \\
f(\infty)=a(\infty)=1 .
\end{gathered}
$$

Eqs.(13) and (14) provide with the quantization of magnetic flux passing through a surface

$$
\int_{\Sigma} \vec{B} \cdot d \vec{\sigma}=\oint_{C} \vec{A} d \vec{l}=n \Phi_{0}, n=1,2,3, \ldots
$$

$\Phi_{0}=\frac{\hbar c}{e}$ is the unit flux quantum. 
Another approach to the vortex problem was formulated in the Ref.[26]. Now we make use of it to consider the Abrikosov vortices taking place in the model (1). To this end, let us introduce the unit vector

$$
n^{a}=\frac{\phi_{a}}{|\phi|}, a=1,2 .
$$

Next it is easily proved that the electromagnetic potentials appearing in Eq.(7) can be expressed as follows

$$
A_{\mu}=\frac{\hbar c}{2 i e} \cdot \frac{1}{\phi^{*} \phi}\left(\phi^{*} \partial_{\mu} \phi-\phi \partial_{\mu} \phi^{*}\right)
$$

Indeed, from

$$
\begin{aligned}
& D_{i} \phi=\partial_{i} \phi-i \frac{e}{\hbar c} A_{i} \phi \\
& \left(D_{i} \phi\right)^{*}=\partial_{i} \phi^{*}+i \frac{e}{\hbar c} A_{i} \phi^{*}
\end{aligned}
$$

we deduce

$$
A_{i}=\frac{\hbar c}{2 i e} \cdot \frac{1}{\phi^{*} \phi}\left[\left(\phi^{*} \partial_{i} \phi-\phi \partial_{i} \phi^{*}\right)-\left(\phi^{*} D_{i} \phi-D_{i}^{*} \phi^{*} \phi\right)\right]
$$

It was shown [27] that the covariant part

$$
\phi^{*} D_{i} \phi-D_{i}^{*} \phi^{*} \phi \sim \partial_{i} \kappa(x)
$$

with $\kappa(x)$ being a function of $x$. Thus this covariant part contributes nothing to the electromagnetic tensor $F_{\mu \nu}$.

Inserting (15) into (16) it is found that

$$
A_{\mu}=\frac{\hbar c}{e} \varepsilon_{a b} n^{a} \partial_{\mu} n^{b} \text { and } F_{\mu \nu}=\partial_{\mu} A_{\nu}-\partial_{\nu} A_{\mu}=2 \frac{\hbar c}{e} \varepsilon_{a b} \partial_{\mu} n^{a} \partial_{\nu} n^{b}
$$

Based on the preceding formulae we get the expression for magnetic field

$$
B_{i}=\frac{1}{2} \varepsilon_{i j k} F_{j k}=\Phi_{0} \frac{1}{2 \pi} \varepsilon_{i j k} \varepsilon_{a b} \partial_{j} n^{a} \partial_{k} n^{b}
$$

Utilizing the results of Ref.[28] Eqs.(14) turns out to be

$$
B_{i}=\Phi_{0} \delta^{2}(\vec{\phi}) D_{i}\left(\frac{\phi}{x}\right)
$$

where $\delta^{2}(\vec{\phi})$ is the two dimensional delta function of two dimensional vector $\vec{\phi}=\left(\phi_{1}, \phi_{2}\right)$ and $D_{i}\left(\frac{\phi}{x}\right)=\frac{1}{2} \varepsilon_{i j k} \varepsilon_{a b} \partial_{j} \phi_{a} \partial_{k} \phi_{b}$

Eq.(18) indicates that there exist the singular points at

$$
\phi_{a}(x, y, z)=0, a=1,2 .
$$

The implicit function theory states that if $D_{i}\left(\frac{\phi}{x}\right) \neq 0$ the general solutions to Eq. (19) can be expressed as

$$
x=x_{i}(s), y=y_{i}(s), z=z_{i}(s), i=1,2, \ldots M .
$$


representing $\mathrm{M}$ isolated singular strings $L_{i}$ with parameters. In the other hand, the theory of delta function gives

$$
\delta^{2}(\vec{\phi})=\sum_{i=1}^{M} \beta_{i} \int_{L_{i}} \frac{\delta^{3}\left(\vec{x}-\vec{x}_{i}(s)\right)}{\left|D\left(\frac{\phi}{u}\right)\right|_{\sum_{i}}}
$$

Here $\sum_{i}$ is the planer element transversal to $L_{i}$ with local coordinates $\left(u_{1}, u_{2}\right)$ and $\beta_{i}$ is the Hopf index of $\phi$-mapping.

Inserting (20) into (18) yields

$$
B_{i}=\Phi_{0} \sum_{j=1}^{M} w_{j} \int_{L_{j}} \frac{d x^{i}}{d s} \delta^{3}\left(\vec{x}-\vec{x}_{j}(s)\right) d s
$$

where $w_{j}=\beta_{j} \eta_{j}, \eta_{j}= \pm 1$ is the Brouwer degree and $w_{j}$ is called the winding number taking the values $\pm n, n=0,1,2, \ldots$.

Finally, it is not difficult to derive from (18) that the magnetic flux is quantized, $\Phi=\int_{\sum} \vec{B} d \vec{\sigma}=\Phi_{0} \sum_{j=1}^{M} w_{j}$

\section{Domain Wall}

In the Section 1 we have noticed that the vacuum manifold $M$ consists of two disconnected points $u_{ \pm}$which are the expectation values of $\phi_{1}$ field, whereas $\left\langle\phi_{2}\right\rangle=0$. This implies that the field $\phi_{1}$ would create a wall separating these two points.

Starting from the Lagrangian (1) we deduce the equation of the domain wall

$$
\begin{array}{r}
\phi_{1}+2 \lambda \phi_{1}\left(\phi^{2}-\beta^{2}\right)=0 \\
\beta^{2}=\frac{\mu^{2}-m^{2}}{\lambda}
\end{array}
$$

Eq. (22) admits the plain wall solution depending upon one coordinate, say, z,

$$
\phi_{1}(z)=\beta \tanh (z / \delta)
$$

which changes from $u_{-}$to $u_{+}$as $\mathrm{z}$ runs from $-\infty$ to $+\infty$. The thickness of the wall is $\delta=\lambda^{-\frac{1}{2}} \beta^{-1}$ and the surface energy density is determined as follows. At first let us calculate the energy-momentum tensor

$$
T_{\mu \nu}=\partial_{\mu} \phi_{1} \partial_{\nu} \phi_{1}-g_{\mu \nu} L
$$

where $g_{\mu \nu}=\operatorname{diag}(1,-1,-1,-1)$ is the metric tensor of the Minkowski space-time. Substituting (23) into (24) gives

$$
T_{0}^{0}=f(z), \quad f(z)=\lambda \beta^{4}\left[\cosh \frac{z}{\delta}\right]^{-4}
$$

The surface energy density reads immediately

$$
\sigma=\int T_{0}^{0} d z=\frac{4}{3} \lambda^{1 / 2} \beta^{3}
$$


It is important to remark that the domain wall is topologically stable. In effect, let us introduce the topological current

$$
j^{\mu}=\varepsilon^{\mu \nu} \partial_{\nu} \phi_{1}
$$

which leads to the conservation of the topological charge

$$
\partial_{\mu} j^{\mu}=0
$$

and the topological charge of the wall is

$$
Q=\frac{1}{\pi} \int_{-\infty}^{+\infty} d z j_{0}=1
$$

\section{CONCLUSION AND OUTLOOK}

In this paper we investigated in detail a model of complex scalar field at finite density. The main results obtained in the foregoing sections are in order:

The spontaneous symmetry breaking occurs only when $\mu=\mu_{\text {critic }}>m$ and the Goldstone theorem is obeyed in broken phase. The boson is condensed and its condensate turns out to be superfluid.

In addition to the above mentioned results the boson condensate behaves as a superconductor under the influence of a magnetic field. This fact is signaled by the existence of the Meissner effect and the Abrikosov votices.

After the spontaneous symmetry breaking realized there appears the domain wall created by the real part of the complex field.

The next step is to consider the theory not only at finite density but also at finite temperature, then we are able to deal with the phase transition of the system. This is a very interesting research problem.

\section{ACKNOWLEDGEMENT}

This paper is financially supported by the Vietnam National Foundation for Science and Technology Development (NAFOSTED).

\section{REFERENCES}

[1] L.D.Landau and E.M.Lifshitz, Statistical Physics, Pergamon Press, NY 1969.

[2] P.G.de Gennes, Superconductivity of Metals and Alloys, W.A.Benjamin, INC. New York - Amsterdam, 1966.

[3] Quang Ho-Kim and Pham Xuan Yem, Elementary Particles and Their Interactions, Pringer, 1998.

[4] C.Itzykson and J.B.Zuber, Quantum Field Theory, McGraw Hill International Editions, 1980.

[5] D.H.Lyth, hep-th $/ 0311040$.

[6] A.Linde, hep-th/0503195.

[7] A.M.Migdal, Sov. Phys. JETP 36 (1973) 1052.

[8] R.F.Sawyer, Phys. Rev. Lett. 29(1972) 382.

[9] D.K.Campell,R.F.Dashen, and J.T.Manassah, Phys. Rev. D12 (1975) 979. ibid D12(1975)1010.

[10] D.B.Kaplan and A.E.Nelson, Phys. Lett. B175 (1986) 57.

[11] M.G.Alford,K.Rajagopal, and F.Wilczek, Nucl. Phys. B537(1999) 443.

[12] J.B.Kogut and D.K.Sinclair, Phys. Rev. D64 (2001) 034508

[13] J.B.Kogut and D.K.Sinclair, Phys.Rev. D70 (2004) 094501 
[14] S.Gupta, hep-lat/0202006.

[15] A.Barducci et al., Phys. Rev. D71 (2005) 016011.

[16] L.He,M.Jin and P.Zhang, Phys. Rev. D71 (2005) 116001.

[17] L.He and P.Zhang, Phys.Lett. B615 (2005) 93.

[18] M.Frank et al., Phys. Lett. B562 ( 2003) 221.

[19] J.O Andersen and T.Brauner, Phys. Rev. D78 (2008) 014030.

[20] O.Scavenius et al., Phys. Rev. C64(2001) 045202

[21] J.O.Andersen, Phys. Rev. D75 (2007) 065011.

[22] M.C.Birse et al., Phys. Lett. B516 (2001) 27.

[23] J.B.Kogut and D. Toublan, Phys. Rev. D64 (2001) 034007.

[24] M.Loewe and C.Villavicencio, Phys. Rev. D70 (2004) 074005.

[25] K.Splittorff et al., Phys. Rev. D64 (2001) 016003.

[26] Yi-Shi Duan et al., cond-mat/010610

[27] L.B.Fu et al., Phys. Rev. D61 (2000) 045004.

[28] Yi-Shi Duan et al., Nucl. Phys. B514 (1998) 705. 